\title{
Influência de estímulos motivacionais no desempenho em testes de salto vertical de atletas de futsal sub-17
}

http://dx.doi.org/10.11606/1807-5509202000040727

\author{
Alan Bruno Silva Vasconcelos* \\ Renata Luiza Farinon** \\ Danton Matheus Perasol** \\ J osé Marcos Melo dos Santos* \\ Victor Hugo de Freitas*
}

*Universidade Federal de Sergipe, Aracaju, SE, Brasil.

**Faculdade de Educação Física, Centro Universitário Integrado, Campo

Mourão, PR, Brasil.

\begin{abstract}
Resumo
0 objetivo deste estudo foi verificar a influência de estímulos motivacionais (encorajamento verbal - EV e estímulo motivacional competitivo - EMC) sobre o desempenho de atletas de futsal em testes de salto vertical. Para isso, foram incluídos neste estudo treze jogadores, do sexo masculino, de uma equipe de futsal Sub-17, com idade média de 16,91 $\pm 1,08$ anos, altura de 1,73 $\pm 4,12 \mathrm{~cm}$ e peso de $71,25 \pm 12,35 \mathrm{~kg}$. Estes jogadores foram submetidos aos testes squat jump (SJ) e countermovement jump (CMJ), executados sem estímulos motivacionais (SM) em um primeiro momento, e executados com estímulos motivacionais (CM) em um segundo momento, respeitando um intervalo de 10 minutos entre os momentos. OS principais resultados encontrados foram que os jogadores apresentaram maior desempenho tanto no SJ (SM 32,11 $\pm 4,95 ;$ CM 35,35 $\pm 4,04 ; P<0,01)$ quanto no CMJ (SM 34,76 \pm $5,25 ; C M 36,51 \pm 4,48 ; P=0,04)$ quando foram realizados estímulos motivacionais. Sendo assim, pode-se concluir que os estímulos motivacionais, sendo eles EV e EMC, ocasionaram melhora no desempenho em testes de SJ e CMJ em jovens jogadores de futsal.
\end{abstract}

Palavras-Chave: Encorajamento verbal; Testes físicos; Performance; Squat jump; Countermovement jump.

\section{Introdução}

A potência de membros inferiores é uma valência física que deve estar bem desenvolvida em atletas de futsal devido a sua importância em momentos decisivos das partidas como nos chutes para o gol, nas disputas de bola, nas mudanças de direçóes e nos contra-ataques. Deste modo, testes de salto vertical (SV), como os propostos por Bosco ${ }^{1}$, Squat jump (SJ) e countermovement jump (CMJ), são frequentemente utilizados para inferir potência de membros inferiores de atleta de futsal ${ }^{2-4}$. Grande parte dos estudos que utilizam esses testes ${ }^{2}$, 5-7 apresentam cuidados metodológicos (equipamento e software utilizado para mensuração, aquecimento, modo de execução, tempo de intervalo, número de tentativas) buscando reduzir vieses em seus resultados. No entanto, o controle sobre os estímulos motivacionais utilizados para que os atletas apresentem melhor desempenho nos testes tem sido negligenciado ou não tem sido relatado ${ }^{2,5-7}$.

Sabe-se que fatores psicológicos podem influenciar o desempenho físico de atletas. Dentre esses fatores, podemos destacar a motivação, que pode ser definida como motivo, seja este interno ou externo, que leva o indivíduo a realizar uma tarefa ${ }^{8}$. Estímulos motivacionais (EM), como o encorajamento verbal, retroalimentaçáo visual e músicas, aplicados antes e durante o exercício podem ocasionar efeitos positivos sobre o desempenho ${ }^{9-13}$. De acordo com Moffatt et al. ${ }^{14}$, o encorajamento verbal (EV) aumentou o consumo máximo de oxigênio $\left(\mathrm{VO}_{2 \max }\right)$, o tempo de corrida, a frequência cardíaca máxima $\left(\mathrm{FC}_{\max }\right)$ e a concentração sanguínea de lactato de não atletas, e aumentou o tempo de corrida e a $\mathrm{FC}_{\max }$ de atletas 
corredores. Além disso, BelkhiriA et al. ${ }^{15}$ reportaram aumento da ativação muscular e da contração voluntária máxima no handgrip quando sujeitos foram submetidos a EV. Esses estudos sugerem queEM, como o EV, devem ser considerados nos protocolos de testes de desempenho físico.

A competitividade é uma características observadas em atletas ${ }^{16}$. Estudos mostram que atletas apresentam maior orientação competitiva (competitividade, orientação para vitória e orientação para metas) comparado com náo atletas ${ }^{16,17}$. Isso sugere que estimular uma competiçâo pelo melhor resultado em testes físicos (estímulo motivacional competitivo EMC), como no teste de SV por exemplo, poderia

\section{Método}

\section{Amostra}

Participaram deste estudo treze jogadores de uma equipe de futsal Sub-17 (sexo masculino, 16,91 \pm 1,08 anos, $173 \pm 4,12 \mathrm{~cm}$ e $71,25 \pm 12,35 \mathrm{~kg}$ ). Essa equipe foi campeã estadual da modalidade na temporada no qual o estudo foi realizado. Foram adotados como critérios de inclusão: treinar regularmente na equipe em questão; ter menos de 17 anos. Como critérios de exclusão foram adotados: lesóes mioarticulares; uso de suplementos alimentares ou medicamentos; doenças no trato respiratório superior; participar também dos treinamentos com a equipe profissional. Este estudo foi aprovado pelo Comitê de Ética em Pesquisa com Seres Humanos do Centro Universitário Integrado (parecer $\mathrm{n}^{\circ} 1.982 .224$ ), respeitando as normas nacionais de biossegurança, e os atletas assinaram um termo de consentimento livre esclarecido aceitando participar do estudo.

\section{Amostra}

Os jovens jogadores foram submetidos a testes de SV, squat jump (SJ) e countermovement jump (CMJ). Esses testes foram aplicados as 16 horas, horário habitual de treinamento da equipe, e com os jogadores sem realizar exercícios por 48-h. Os testes de SV foram executados sem estímulos motivacionais (SM) e com estímulos motivacionais (CM). Em um primeiro momento (executados SM) os atletas foram mantidos afastados da área de testes e se apresentavam individualmente para ser uma maneira de motivar o empenho dos atletas durante o teste. No entanto, até o momento, não foram encontrados estudos investigando o efeito de EM, sejam eles EMC ou EV no desempenho em testes de SV de jogadores de futsal. Desse modo, na tentativa de reduzir vieses nos resultados encontrados em testes de SV, uma vez que a padronização dessa variável é negligenciada ${ }^{5-7,18}$, o presente estudo tem como objetivo verificar a influência de EM, (EV e EMC) sobre o desempenho de atletas de futsal em testes de SV. Os resultados encontrados servirão como informação relevante para profissionais que trabalham com testes de SV para inferir potência de membros inferiores de atletas.

realiza-los. Os saltos foram realizados separadamente para que nenhum jogador soubesse o resultado do outro jogador. Além disso, os resultados dos testes foram ocultados, e os atletas não foram informados dos resultados desempenhados. Os jogadores foram orientados sobre a execução dos testes e que deveriam saltar o mais alto possível. No entanto, durante a execução dos testes, nenhum EM foi dado aos jogadores (sem EV e EMC). Após 10 minutos de intervalo, todos os 13 jogadores se apresentaram em conjunto para realizar os testes de SV e foram estimulados a competir entre eles para conseguir o melhor desempenho (foi realizado EMC). Neste momento, os jogadores receberam $\mathrm{EV}$ antes de cada tentativa, e foram informados sobre os resultados desempenhados em cada salto, servindo de parâmetro para uma tentativa melhor. Um aquecimento prévio (realizado individualmente) consistindo de trotes, salto unilateral, deslocamentos laterais e alongamento dinâmico foram realizados por 5 minutos. Após o aquecimento, os jogadores realizaram 2 SJ e 2 CMJ submáximos, os quais serviram como saltos de aquecimento e de familiarização. 3 minutos após os saltos de aquecimento, os testes foram iniciados ( $\mathrm{SM} \mathrm{e}$ $\mathrm{CM}$ ). Vale ressaltar que testes de $\mathrm{SV}$ são rotineiramente realizados por esses jogadores como parte da avaliação física realizada pela equipe técnica da equipe.

\section{Testes de salto vertical}

Os testes de SJ e CMJ foram utilizados para inferir a potência de membros inferiores. Para 
isso, foi utilizado um tapete de contato (Cefise ${ }^{\circledast}$, Brasil) e os resultados foram obtidos com por meio de um software (Cefise ${ }^{\varpi}$, Brasil). Para o teste SJ, os jogadores partiram de um agachamento com uma flexão do joelho de aproximadamente $90^{\circ}$, e realizaram o salto com as mãos apoiadas na cintura. Já para o CMJ, os jogadores partiram da posição ortostática, realizaram um agachamento com ciclo alongamento-encurtamento, e logo em seguida, realizaram um salto. Para cada teste foram permitidas 3 tentativas, respeitando 1 min de intervalo entre elas, e a maior altura alcançada nas tentativas foi registrado como o desempenho dos jogadores.

\section{Estatística}

A normalidade dos dados foi testada por meio do teste de Shapiro-Wilk. Para comparar o desempenho nos testes de SJ e CMJ realizados CM e SM foi utilizado o Test-t para amostras dependentes. O software utilizado foi o Statistica (v.8.0, StatSoft ${ }^{\oplus}$, Tulsa, Ok) considerando diferença o valor de $p<0,05$. A inferência prática baseada em magnitude foi utilizada para identificar a chance das diferenças no desempenho nos testes de SV realizados CM e SM serem diferentes da mínima mudança detectável (MMD - 0.2 multiplicado pelo desvio padrão inicial). Nesta análise foi considerado um intervalo de confiança de $90 \%$. As mudanças foram avaliadas qualitativamente como: < $1 \%$ quase certamente não; $1-5 \%$ muito improvável; 5-25\% improvável; 25-75\% possível; 75-95\% provável; $95-99 \%$ muito provável e > 99\% quase certamente sim. Quando os valores negativos e positivos apresentaram resultados > $5 \%$, a inferência foi considerada inconclusiva. As análises foram realizadas nas tabelas disponíveis em http://www.sportsci.org/.

\section{Resultados}

O desempenho nos testes SJ (SM: 32,11 14,95 ; CM: 35,35 \pm 4,04; $\mathrm{p}<0,01)$ e CMJ (SM 34,76 \pm 5,25; CM 36,51 \pm 4,48; $\mathrm{p}=0,04)$ foram maiores quando realizado com EM comparado com quando não ocorreram EM (FIGURA 1).
A inferência prática baseada em magnitude mostrou que é muito provável (SJ) e provável (CMJ) que os jogadores apresentem maior desempenho quando os testes SJ e CMJ são executados com EM (SJ: 99/1/0; CMJ: 79/21/0; FIGURA 2).

FIGURA 1 - - Desempenho no Squat jump (SJ ) e no contermovement J ump (CMJ ) de jogadores de futsal sem (barras pretas) e com (barra cinza) estímulos motivacionais.)mulos motivacionais.
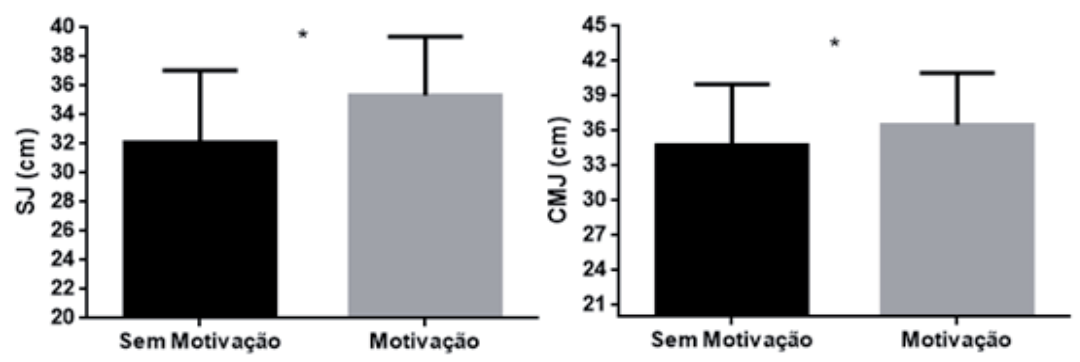
FIGURA 2 - Diferença (cm) individual da altura alcançada nos testes de squatjump (A) e contermovement jump (B).

A linha tracejada demarca a mínima mudança detectável $(0,2 \times \mathrm{DP})$, que na FIGURAA foi de $-1,06$ e 1,06 e na FIGURA B foi $-1,12$ e 1,12 .
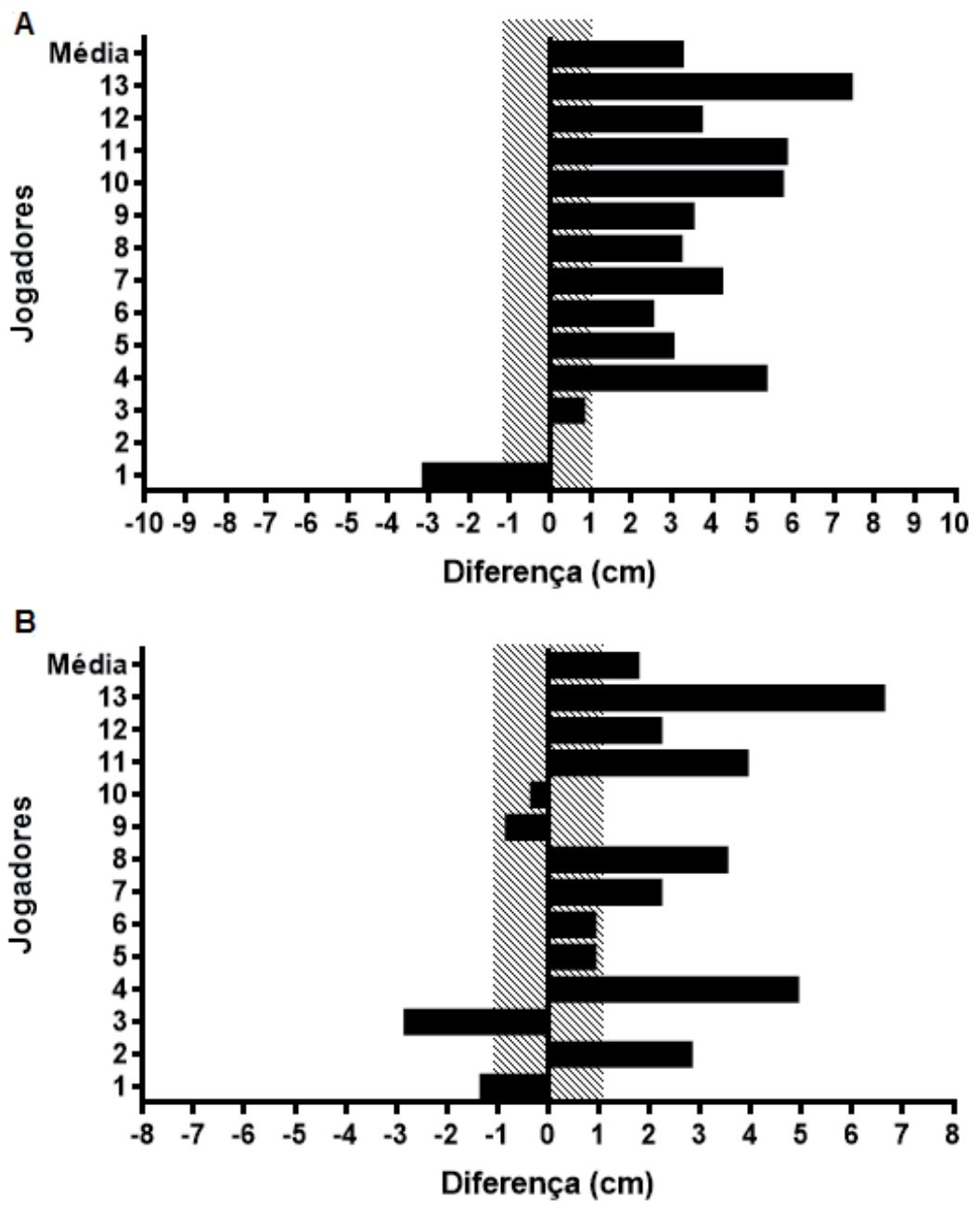

\section{Discussão}

Com o objetivo de avaliar a influência de EM no desempenho em testes de SV de jovens jogadores de futsal, os resultados encontrados no presente estudo mostraram que os jogadores obtiveram desempenho superior em testes de SJ e CMJ após receberem EM (EV e EMC).

A hipótese levantada no presente estudo para explicar um maior desempenho nos testes de SJ e $\mathrm{CMJ}$ perpassam as perspectivas psicológicas que se baseiam na presença de estímulos motivacionais positivos, que refletiram em uma maior ativação de fibras musculares e consequentemente maior potência de membros inferiores. Estudos demonstram que sujeitos que receberam EM apresentaram maior ativação muscular, avaliado por eletromiografia, e maior desempenho em testes de força ${ }^{15,19}$. De acordo com Pessota et al. ${ }^{20}$, a motivação possibilita um aumento na carga de trabalho em decorrência de reaçôes psicofísicas, que de algum modo, se relacionam com a redução subjetiva do esforço e o aumento dos estados afetivos. Desse modo, estímulos motivacionais podem ser utilizados como estratégia para a melhoria do desempenho físico, sendo a motivação vista como um recurso ergogênico sem efeitos colaterais e lícito.

Outros estudos corroboram os resultados encontrados no presente estudo no tocante a influência do EV em melhorar o desempenho 
em testes físicos 14, 15, 21. KARABA-JAKOVLJEviĆ et al., ${ }^{21}$ por exemplo, demonstraram aumento no desempenho no teste de Wingate de indivíduos que receberam EV. No entanto, cabe salientar que alguns estudos não reportaram efeitos positivos de EM para aumentar o desempenho em testes físicos ${ }^{22}$. Esses resultados conflitantes sustentam a importância dos resultados encontrados no presente estudo, os quais, sugerem que EM podem influenciar nos resultados encontrados em testes de SV.

Embora a amostra do presente estudo seja composta por jovens jogadores de futsal (categoria sub-17), os efeitos ergogênicos de EM podem não estar relacionados ao tempo ou status de treinamento. Amagliani et al. ${ }^{23}$

demonstraram que mulheres treinadas e não treinadas são igualmente influenciadas por EM. Sendo assim, apesar da necessidade de outros estudos com jogadores de futsal de outras categorias, até o momento, os resultados do presente estudo encorajam uma padronização quanto aos EM realizados durante testes de $\mathrm{SV}$ realizados em jogadores de futsal, independente da categoria.

Conclui-se que estímulos motivacionais, sendo eles encorajamento verbal e estímulo motivacional competitivo proporcionam aumento no desempenho de jovens atletas de futsal nos testes SJ e CMJ. Esses resultados sugerem que profissionais que utilizam SV como testes para avaliar o desempenho físico em sua rotina de trabalho ou em pesquisas devem controlar com maior rigor fatores que podem influenciar a motivação dos jogadores durante os testes. Estimular uma competição pelo melhor resultado, mostrar o resultado de cada salto, e realizar constante EV podem ser estratégias utilizadas para que os atletas apresentem maior desempenho nesses testes (CMJ e SJ). Independente do EM utilizado, a padronização desses estímulos parecem ser importantes para evitar resultados enviesados que possam comprometer a avaliação do desempenho.

\title{
Conflito de interesses
}

Os autores declaram não haver conflito de interesse que poderia se constituir em um impedimento para a publicação.

\begin{abstract}
Influence of verbal and competitive encouragement on performance in vertical jump tests of U-17 futsal athletes.

The purpose of this study was to verify the influence of motivational stimuli (verbal encouragement - EV, and competitive motivational stimulus - EMC) on the performance of futsal athletes in vertical jump tests. For this purpose, thirteen male players of an U17 futsal team participated in this study, with a mean age of $16.91 \pm 1.08$ years, height of $1.73 \pm 4.12 \mathrm{~cm}$, and weight of $71.25 \pm 12.35 \mathrm{~kg}$. The players executed the squat jump (SJ) and vertical jump with countermovement (CMJ), performed without motivational stimuli (MS) in the first moment, and with MS in the second moment, respecting a 10 min interval between moments. The principal results demonstrated that players presented higher performance in both the SJ (SC $32.11 \pm 4.95$, C $35.35 \pm 4.04, P<0.01)$ and CMJ (SC $34.76 \pm 5.25, C 36.51 \pm 4.48, P=0.04)$ when motivational stimuli were provided. Therefore, it can be concluded that motivational stimulus, either EV or EMC, improved the performance of young athletes in $\mathrm{SJ}$ and $\mathrm{CMJ}$ jump tests.
\end{abstract}

KeYwords: Verbal encouragement; Physical tests; Performance; Squat jump; Countermovement jump. 


\section{Referências}

1. Bosco C. La valutazione della forza con il test di Bosco. 2 ed. Roma: Società Stampa Sportiva; 2002.

2. Freitas VH, de Souza EA, Oliveira RS, Pereira LA, Nakamura FY. Effect of four successive days of games in muscle power, perceived stress and recovery in futsal players. Rev Bras Educ Fís Esporte. 2014.

3. Freitas VH, Miloski B, Bara-Filho MG. Quantification of training load using session RPE method and performance in futsal. Revista Brasileira de Cineantropometria \& Desempenho Humano. 2012;14(1):73-82.

4. Miloski B, de Freitas VH, Nakamura FY, de ANFC, Bara-Filho MG. Seasonal training load distribution of professional futsal players: effects on physical fitness, muscle damage and hormonal status. J Strength Cond Res. 2016;30(6):1525-33.

5. Johnston RD, Gibson NV, Twist C, Gabbett TJ, MacNay SA, MacFarlane NG. Physiological responses to an intensified period of rugby league competition. J Strength Cond Res. 2013;27(3):643-54.

6. Delextrat A, Trochym E, Calleja-Gonzalez J. Effect of a typical in-season week on strength jump and sprint performances in national-level female basketball players. J Sports Med Phys Fitness. 2012;52(2):128-36.

7. Freitas VH, Nakamura FY, Miloski B, Samulski D, Bara-Filho MG. Sensitivity of physiological and psychological markers to training load intensification in volleyball players. J Sports Sci Med. 2014;13(3):571-9.

8. Samulski D. Psicologia do esporte. São Paulo: Manole; 2002.

9. Nakamura PM, Pereira G, Papini CB, Nakamura FY, Kokubun E. Effects of preferred and nonpreferred music on continuous cycling exercise performance. Percept Mot Skills. 2010;110(1):257-64.

10. Szmedra L, Bacharach DW. Effect of music on perceived exertion, plasma lactate, norepinephrine and cardiovascular hemodynamics during treadmill running. Int J Sports Med. 1998;19(1):32-7.

11. Eliakim M, Bodner E, Eliakim A, Nemet D, Meckel Y. Effect of motivational music on lactate levels during recovery from intense exercise. J Strength Cond Res. 2012;26(1):80-6.

12. Andreacci JL, LeMura LM, Cohen SL, Urbansky EA, Chelland SA, Von Duvillard SP. The effects of frequency of encouragement on performance during maximal exercise testing. J Sports Sci. 2002;20(4):345-52.

13. Figoni SF, Morris AF. Effects of Knowledge of Results on Reciprocal, lsokinefk Strength and Fatigue. J Orthop Sports Phys Ther. 1984;6(3):190-7.

14. Moffatt RJ, Chitwood LF, Biggerstaff KD. The influence of verbal encouragement during assessment of maximal oxygen uptake. J Sports Med Phys Fitness. 1994;34(1):45-9.

15. Belkhiria C, de Marco G, Driss T. Effects of verbal encouragement on force and electromyographic activations during handgrip exercise. J Sports Med Phys Fitness. 2017.

16. Gill DL, Dzewaltowski DA, Deeter TE. The relationship of competitiveness and achievement orientation to participation in sport and nonsport activities. Journal of Sport and Exercise Psychology. 1988;10:139-50.

17. Gill DL, Kelley BC, Martin JJ, Caruso CM. A Comparison of Competitive-Orientation Measures. J Sport Exerc Psychol. 1991;8:266-80.

18. Midgley AW, Marchant DC, Levy AR. A call to action towards an evidence-based approach to using verbal encouragement during maximal exercise testing. Clin Physiol Funct Imaging. 2017.

19. Binboga E, Tok S, Catikkas F, Guven S, Dane S. The effects of verbal encouragement and conscientiousness on maximal voluntary contraction of the triceps surae muscle in elite athletes. J Sports Sci. 2013;31(9):982-8.

20. Pessota V, Barreto AT, De Mello MT, Antunes HKM. Influência dos aspectos motivacionais sobre as respostas afetivas após teste de esforço máximo. Rev Psicol Saúde. 2011;3(2):1-12.

21. Karaba-Jakovljevic D, Popadic-Gacesa J, Grujic N, Barak O, Drapsin M. Motivation and motoric tests in sports. Med Pregl. 2007;60(5-6):231-6.

22. Bullinger DL, Hearon CM, Gaines SA, Daniel ML. Concurrent verbal encouragement and wingate anaerobic cycle test performance in females: athletes vs. non-athletes. Intern J Exerc Sci. 2012;5(3):239-44.

23. Amagliani RM, Peterella JK, Jung AP. Type of encouragement influences peak muscle force in college-age women. Intern J Exerc Sci. 2010;3(4):165-73. 
ENDEREÇO Alan Bruno da Silva Vasconcelos Av. Marechal Rondo, s/n 49030-730 - Aracaju - SE - Brasil E-mail: abs.vasconcelos@gmail.com

Submetido: 12/01/2018

Revisado: 01/ 10/2018

Aceito: 21/ 04/ 2019 